\title{
Premiers résultats à propos du diagnostic sérologique de la bilharziose par immuno-fluorescence sur coupes à la congélation de Scbistosoma baematobium
}

\author{
par Michel-André POTHIER et Maria de LOURDES-SAMPAIO-XAVIER
}

[Travail réalisé en collaboration

avec la Chaire d'Entomologie et d'Helminthologie de l'Ecole Nationale de Santé Publique et de Médecine Tropicale de Lisbonne (Portugal) ( $\mathrm{P}^{r}$ J. Fraga de Azevedo), et l'Institut de Médecine et d'Hygiène Tropicales de la Faculté mixte de Médecine et de Pharmacie de 69-Lyon (France) ( $\mathrm{P}^{\mathrm{r}} \mathrm{J}$. COUDERT)]

\section{Résumé}

On observe une très importante différence quantitative entre les titres obtenus pour chacun des types de bilharzioses dans le diagnostic sérologique par immunofluorescence.

Cette différence est sans doute à rapporter en partie à une question de spécificité antigénique; notre antigène est constitué par des coupes de Schistosoma haematobium avec lesquelles des sérums homologues sont ceux qui réagissent le plus intensément. Nos premiers résultats ne portent que sur l'analyse des sérums en présence de l'antigène Schistosoma haematobium et devra être complété par l'étude en parallèle avec l'antigène Schistosoma mansoni.

\section{Summary}

The apnlication of Immunofluorescence to the serological diagnosis of human bilharziasis let appear an important quantitative difference between the titles obtained in each type of Schistosomiasis.

This difference partly depends on the antigenic specificity.

The antigens here used are sections of Schistosoma haematobium on which the homologous sera are those which react the most intensively.

The first results given in this work only concern sera confronted with $S$. haematobium antigen; they need to be completed by a parallel study with $S$. mansoni antigen. 
L'application de l'immuno-fluorescence au diagnostic sérologique des bilharzioses humaines a donné lieu à un certain nombre de travaux.

Dès 1966, nous avons mis au point le séro-diagnostic de la bilharziose par une nouvelle technique d'immuno-fluorescence sur coupes à la congélation de Schistosoma mansoni en souhaitant pouvoir effectuer cette réaction à partir de Schistosoma haematobium ou de Schistosoma japonicum, employés comme antigène.

En effet, ceci permettrait facilement l'étude sérologique des bilharzioses urinaires ou extrême-orientales avec un antigène homologue, ce qui, en pratique n'a jamais pu être réalisé jusqu'ici.

Nous basant sur l'expérience acquise, nous avons mis au point, pour la bilharziose, une technique d'immuno-fluorescence sur coupes à la congélation de Schistosoma haematobium, dont nous présentons ici une première série de résultats.

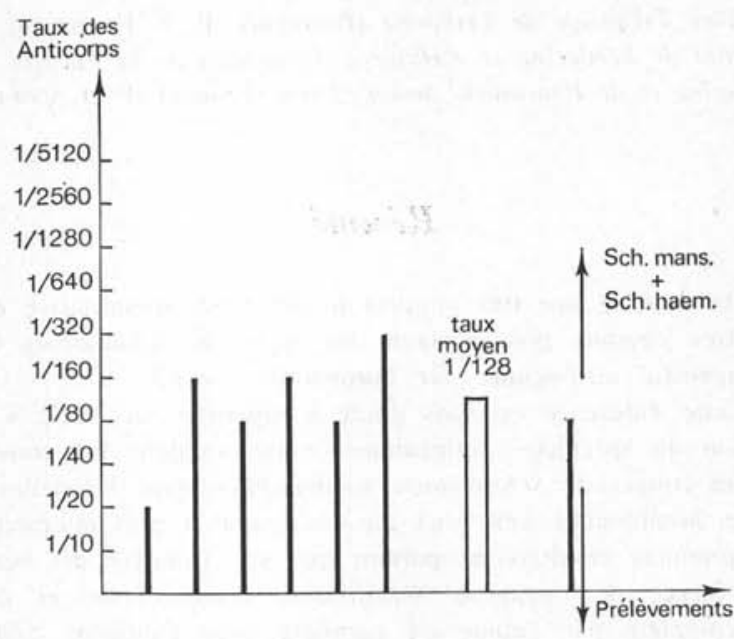

TABleau I. - Séro-diagnostic de la bilharziose à Schistosoma mansoni par Immuno-fluorescence sur coupes de Schistosoma haematobium adultes

\section{I. - Matériels et méthodes}

\section{a) L'antigène.}

L'antigène est constitué par des Schistosoma haematobium adultes, obtenus par l'entretien au laboratoire de la souche de Guinée Portugaise sur hamster.

Ces parasites sont recueillis, puis lavés à trois reprises dans du sérum physiologique à $\mathrm{pH} 7,2$.

Un couple de Schistosomes est enroulé dans un fragment de muscle strié de cobaye sain (muscle de la paroi abdominale). Le cylindre ainsi réalisé est congelé à $-20^{\circ} \mathrm{C}$, puis coupé au cryostat à $4 \mu$.d'épaisseur. 


\section{b) Mode opératoire et lecture des résultats.}

Les modalités techniques de la réaction d'immuno-fluorescence indirecte sur coupes de Schistosomes ont déjà été précisées dans des publications antérieures.

Une contre-coloration par le bleu d'Evans permet de masquer les fluorescences non spécifiques des éléments parasitaires.

Une réaction positive se traduit par une vive fluorescence jaune-verte des Schistosomes, tandis qu'une réaction négative s'objective par une coloration rouge des vers qui prennent la même teinte que le muscle strié servant de support (photos I, II).

Sur le p'an pratique, cette technique présente les mêmes avantages que ceux décrits au sujet de Schistosoma mansoni.

\section{II. - Résultats et commentaires}

Au total, nous avons effectué des examens sérologiques, dont certains correspondent à des contrôles de spécificité, et d'autres à des réactions pratiquées chez des malades bilharziens.

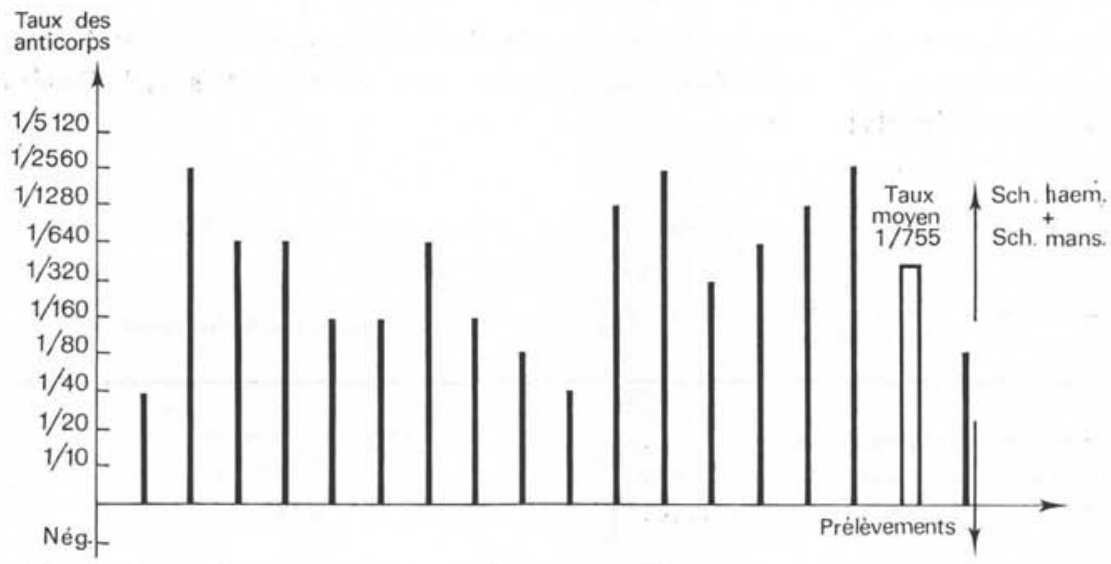

TABleAU II. - Séro-diagnostic de la bilharziose à Schistosoma haematobium par Immunofluorescence sur coupes de Schistosoma haematobium adultes

\section{a) Contrôles de spécificité.}

La spécificité de la réaction a été vérifiée par des examens de contrôle, effectués chez des sujets sains ou chez des malades atteints de diverses affections parasitaires ou non.

Il s'agit de :

- Sujets sains

19 sérums

- Parasitoses:

- Protozooses 16 sérums 
- Helminthiases à vers ronds ........... 19 sérums

- Helminthiases à vers plats $\ldots \ldots \ldots \ldots \ldots .21$ sérums

- Mycoses:

- Candidoses viscérales ............... 3 sérums

- Maladies infectieuses:

- Syphilis ...................... 5 sérums

Ces sérums étudiés sur des coupes à la congélation de Schistosoma haematobium, ont été négatifs dès la dilution de $1 / 10$, sauf :

- pour un sérum de Maladie du Sommeil, dont la possibilité d'une bilharziose associée n'était pas écartée,

- de même que pour un sérum de Paludisme,

- pour un sérum de Distomatose dont la lecture de la première dilution était douteuse.

\section{b) Valeur diagnostique.}

La valeur diagnostique de cette technique d'immuno-fluorescence peut être appréciée à partir d'une série préliminaire de sérologies dont les résultats sont indiqués dans les tableaux suivants:

TABLEAU III. - Comparaison des taux moyens obtenus avec les divers types de bilharzioses

\begin{tabular}{||c|c||}
\hline Antigène & \\
\hline Sérums & Schistosoma haematobium \\
\hline Schistosoma haematobium $\ldots \ldots \ldots \ldots$ & $1 / 755$ \\
Schistosoma mansoni $\ldots \ldots \ldots \ldots \ldots$ & $1 / 128$ \\
Schistosoma haematobium $\ldots \ldots \ldots \ldots$ & $1 / 80$ \\
$+\ldots$ & \\
Schistosoma mansoni $(1$ cas) $\ldots \ldots \ldots$ & \\
\hline
\end{tabular}

\section{Bibliographie}

Lourdes Sampaio Xavier (Maria de), Fraga de Azevedo (Joao) et Avelino (Ilda), 1968. Importance d'Oscillatoria formosa Bory dans la culture au laboratoire des mollusques vecteurs du Schistosoma haematobium. Bull. Soc. Path. Exot., 61: 52-66.

Pothier (Michel-André), 1967. Le sérodiagnostic de la bilharziose par une nouvelle technique d'Immuno fluorescence sur coupe à la congélation de Schistosoma mansoni (Résultats de 773 examens). Thèse Médecine, Paris, 160 p., 185 réf. 